\title{
Correction
}

\section{Correction: Kanie, N., et al. Integration and Diffusion in Sustainable Development Goals: Learning from the Past, Looking into the Future. Sustainability 2014, 6, 1761-1775}

\section{Norichika Kanie ${ }^{1,2, *}$, Naoya Abe ${ }^{3}$, Masahiko Iguchi ${ }^{1}$, Jue Yang ${ }^{4}$, Ngeta Kabiri ${ }^{2}$, Yuto Kitamura $^{5}$, Shunsuke Managi ${ }^{4}$, Ikuho Miyazawa ${ }^{6}$, Simon Olsen ${ }^{6}$, Tomohiro Tasaki ${ }^{7}$, Taro Yamamoto ${ }^{8}$, Tetsuro Yoshida ${ }^{6}$ and Yuka Hayakawa ${ }^{1}$}

1 Department of Value and Decision Science, Graduate School of Decision Science and Technology, Tokyo Institute of Technology, 2-12-1-W9-43 Ookayama, Meguro-ku, Tokyo 152-8552, Japan; E-Mails: miguchi@valdes.titech.ac.jp (M.I.); haya@valdes.titech.ac.jp (Y.H.)

2 The United Nations University Institute of Advanced Studies (UNU-IAS), Pacifico-Yokohama, 1-1-1 Minato Mirai, Nishi-ku, Yokohama 220-8502, Japan; E-Mail: kabiri@ias.unu.edu

3 Department of International Development Engineering (IDE), Graduate School of Science and Engineering, Tokyo Institute of Technology, 14-4, 2-12-1, Ookayama, Meguro-ku, Tokyo 152-8552, Japan; E-Mail: nabe@ide.titech.ac.jp

4 Graduate School of Environmental Studies, Tohoku University, 6-6-20 Aramaki-Aza-Aoba, Aoba, Sendai 980-8579, Japan; E-Mails: yang.jtohoku@gmail.com (J.Y.); managi@mail.kankyo.tohoku.ac.jp (S.M.)

5 Graduate School of Education, The University of Tokyo, 7-3-1 Hongo, Bunkyo-ku, Tokyo 113-0033, Japan; E-Mail: yuto.kitamura@gmail.com

6 Integrated Policies for Sustainable Societies Area, IGES 2108-11 Kamiyamaguchi, Hayama, Kanagawa 240-0115, Japan; E-Mails: miyazawa@iges.or.jp (I.M.); olsen@iges.or.jp (S.O.); t-yoshida@iges.or.jp (T.Y.)

7 National Institute for Environmental Studies (NIES), 16-2 Onogawa, Tsukuba-City, Ibaraki 305-8506, Japan; E-Mail: tasaki.tomohiro@nies.go.jp

8 Department of International Health, The Institute of Tropical Medicine, Nagasaki University, 1-12-4 Sakamoto, Nagasaki 852-8523, Japan; E-Mail: y-taro@nagasaki-u.ac.jp

* Author to whom correspondence should be addressed; E-Mail: kanie@valdes.titech.ac.jp or kanie@ias.unu.edu; Tel./Fax:+81-3-5734-2189. 
The authors wish to make the following correction, due to a typographical error, to this paper [1]. On page 1761, the author name "Shunsuke Mangagi" should be "Shunsuke Managi".

The authors would like to apologize for any inconvenience caused to the readers by this change.

\section{Reference}

1. Kanie, N.; Abe, N.; Iguchi, M.; Yang, J.; Kabiri, N.; Kitamura, Y.; Mangagi, S.; Miyazawa, I.; Olsen, S.; Tasaki, T.; et al. Integration and Diffusion in Sustainable Development Goals: Learning from the Past, Looking into the Future. Sustainability 2014, 6, 1761-1775.

(C) 2014 by the authors; licensee MDPI, Basel, Switzerland. This article is an open access article distributed under the terms and conditions of the Creative Commons Attribution license (http://creativecommons.org/licenses/by/3.0/). 